\title{
Byzanz in der geistigen Auseinandersetzung mit den \\ Osmanen \\ Ein Überblick
}

\author{
Peter Schreiner
}

Die über neun Jahrhunderte andauernde politische und geistige Auseinandersetzung zwischen Byzanz und der muslimischen Welt hat sicher im 14. und 15. Jahrhundert auch in der theoretischen Diskussion ihren stärksten Niederschlag gefunden. Obwohl die zugrunde liegenden Texte, auf die unten näher einzugehen ist, schon seit geraumer Zeit und zum Teil in guten Ausgaben und sogar Übersetzungen erreichbar sind, bedurfte es der Rede von Papst Benedikt XVI. an der Regensburger Universität im Jahr 2006, ehe die evidentermaßen noch heute gültige Brisanz des philosophisch-theologischen Gedankengutes aus Byzanz einer breiteren Öffentlichkeit bekannt und bewusst wurde. Der Papst zitierte aus dem 7. Teil des Dialogs Kaiser Manuels mit einem Perser, der im Verlauf dieses Beitrags noch ausführlicher zu behandeln ist, Passagen zur kriegerischen Kampfbereitschaft des Islam in Glaubensfragen. ${ }^{1}$

Es ist nicht Ziel dieser knappen Untersuchung, die ausgewählten Zitate aus der Schrift Kaiser Manuels im Zusammenhang mit der Rede des Papstes erneut zu interpretieren. Dies ist inzwischen von verschiedenen Standpunkten her geschehen. ${ }^{2}$ Die Zitate sind in ihrem historischen Kontext, der in der öffentlichen Diskussion überwiegend unbeachtet blieb, nicht nur Ausdruck der byzantinischen Theologie, die den heiligen Krieg ebenso wie den Kreuzzug nicht kennt. ${ }^{3}$ Sie sind auch Zeichen der Verzweiflung eines Kaisers, der vom Sultan zur Kriegsfolge gezwungen wurde und keine eigenen Waffen, sondern nur den diplomatischen Dialog der eigenen Vernunft zur Verfügung hat. Es geht in den

1 Benedikt XVI., 2006, 12-32.

2 Zunächst geschah dies in der oben genannten Schrift, teilweise in wenig kompetenter Form und unter Zugrundelegung heutiger politologischer und gesellschaftspolitischer Aspekte (S. 33-133), sowie in den Akten eines Colloquiums, das den byzantinischen Ausgangspunkt kaum mehr erkennen lässt (Luthe 2008). Den besten Beitrag zu dieser Fragestellung und eine ernsthafte theologische Untersuchung der von Papst Benedikt zitierten Textstellen, die an verschiedenen Ansichten des Kaisers auch Kritik übt, bringt Demetracopoulos 2008.

3 Dieser affirmative Satz ist in der byzantinistischen Forschung nicht unumstritten, und es wird auf Vorbereitungen und Handlungen in einigen kriegerischen Unternehmungen hingewiesen, die diese unter den Schutz Gottes zu stellen scheinen; vgl. die Darstellung von Kolia-Dermatzaki 1991. Eine weitaus größere Rolle spielt in Byzanz die Idee vom gerechten Krieg, vgl. Stouraitis 2009. 
folgenden Ausführungen also darum, die geistige Auseinandersetzung mit den Osmanen in ihrer historischen Entwicklung zu betrachten.

\section{Die politische und territoriale Annäherung der Osmanen an}

\section{Byzanz}

Wenngleich dieser Beitrag nicht der politischen Auseinandersetzung zwischen Byzanz und den Osmanen gewidmet ist, sondern der geistigen, so ist ein knapper Überblick über die historischen Geschehnisse deshalb notwendig, weil sich die osmanische Bedrohung grundsätzlich von der anderer Feinde des byzantinischen Reiches, früherer oder auch gleichzeitiger, unterscheidet. Dieser Unterschied ist von zwei zentralen Phänomenen geprägt: Die Osmanen standen zum einen durch ihren muslimischen Glauben in einem viel tiefer greifenden Gegensatz zu den Byzantinern als christliche Feinde des byzantinischen Reiches, und es gelang ihnen zum andern, das Reich, sowohl von der kleinasiatischen wie der europäischen Seite her, einzukreisen und diesen militärischen Ring immer enger an Konstantinopel heranzuziehen. Einige Vergleichsbeispiele sollen die nunmehr neue Situation im Gegensatz zu früheren Angriffen von andersgläubigen Völkern veranschaulichen.

1. In den ersten drei Jahrzehnten des 7. Jahrhunderts erreichen sasanidische Truppen das Marmarameer und den Bosporus. Der im Jahr 626 gescheiterte Zusammenschluss von Awaren und Sasaniden machten die Bedrohung trotzdem zu einem nur punktuellen Ereignis. Der Sieg des Kaisers Herakleios über die Sasaniden im folgenden Jahr ließ diese als politische und ideologische Gegner für Byzanz ausscheiden.

2. Die seit 640 regelmäßigen Feldzüge der Araber haben zu keiner dauernden Ansiedlung diesseits der Euphratgrenze geführt, während Belagerungen Konstantinopels $(674-678,717)$ mit verschiedenen Mitteln abgewehrt werden konnten. Gemeinsame militärische Aktionen der Bulgaren im Balkanraum und der Araber kamen nicht zustande.

3. Erstmals im 11. Jahrhundert entstand, nach der Schlacht von Mantzikert (1071), im seldschukischen Sultanat von Rum ein islamischer Staat auf byzantinischem Territorium, der am Ende des Jahrhunderts mit der (kurzzeitigen) Verlegung des Regierungssitzes nach Nikaia in bedenkliche Nähe zu Konstantinopel gerückt war. Auf der anderen Seite verfügte Byzanz aber, nach dem Sieg über die Bulgaren (1014) und der Annektierung des bulgarischen Reiches über eine sichere Operations- und Versorgungsbasis im europäischen Raum. Die ersten beiden Kreuzzüge, deren politische (und mi- 
litärische) Bedeutung für Byzanz unterschiedlich diskutiert werden, haben mit hoher Wahrscheinlichkeit zumindest eine Konsolidierung der seldschukischen Macht in den küstennahen Regionen des byzantinischen Reiches, besonders am Marmarameer verhindert. ${ }^{4}$

Als 1261 Kaiser Michael Palaiologos Konstantinopel zurückgewann, zwang ihn die Bedrohung westlicher Mächte, die kleinasiatischen Provinzen militärisch zu vernachlässigen. Diese schienen zudem auch deshalb relativ gesichert, als sich (nach 1243) unter dem Druck der mongolischen Ilkane das seldschukische Reich aufzulösen begann. Gleichzeitig aber, von der byzantinischen Politik unbemerkt oder in den möglichen Folgen falsch eingeschätzt, erfolgte die Verselbständigung und Zuwanderung turkmenischer Stämme bis an die Grenzen der Kleinasien umgebenden Meere. ${ }^{5}$ Erstmals zum Jahre 1301 erwähnen byzantinische Quellen den Namen „Atman“ als Anführer eines turkmenischen Stammes, der damals schon länger im bithynischen Berg- und Hügelland ansässig war, ohne bisher durch besondere kriegerische Aktionen aufgefallen zu sein. ${ }^{6}$ Es ist als Faktum festzuhalten, dass zwischen 1301 und 1331 die gesamte kleinasiatische Küstenregion des Marmarameeres in osmanische Hand fiel. Für die Konsolidierung des osmanischen Staates und den Übergang von halbnomadischer zu sesshafter Lebensweise ist von besonderer Bedeutung, dass jene Region nicht nur ausnehmend reich an städtischem Leben war, sondern bis 1261 als Kernzone des „Nikänischen Staates“ eine besondere wirtschaftliche und kulturelle Förderung erfahren hatte, deren Abglanz auch zu Beginn des 14. Jahrhunderts noch nicht ganz erloschen war. ${ }^{7}$ Während am Ende des 11. Jahrhundert ein seldschukischer Herrschersitz Nikaia eine ephemere Erscheinung geblieben war, war das nahe gelegene Bursa seit 1327 bis zur Übersiedlung nach Adrianopel (nach 1369) fester Sitz des Sultans. Zu Beginn des 8. Jahrzehnts (seit dem Fall der Festung Gallipoli im Jahr 1354) geriet auch der jenseits der Meerenge liegende thraki-

4 Die negative Haltung der byzantinischen Politik zur Kreuzzugsbewegung und zur Durchführung dieser Unternehmungen geht aus einer Fülle von Quellenstellen hervor und ist in der Forschung unbestritten. Es ist aber bisher zu wenig in Betracht gezogen worden, inwieweit die Kreuzzüge nicht doch weitere seldschukische Angriffe in die Küstenregionen des byzantinischen Reiches durch eine „Ablenkungstaktik“ unterbunden haben und somit indirekt für das byzantinische Reich hilfreich waren.

5 Werner 1966, 98-115 („Niedergang und Aufstieg: die anatolischen beylikler“) hat diese Vorgänge herausgearbeitet.

6 Inalcik 1993, 77-99 betont noch, vor allem auf der Basis (späterer) osmanischen Quellen den kriegerischen Charakter des Vordringens im 13. Jh., während Hopwood 1992, 151155 auf die eher friedliche Niederlassung eines halbnomadischen Volkes in Bithynien hinweist.

7 Für die Konsolidierung der osmanischen Macht gerade in dieser geographischen Zone hat Werner 1966, 115 eine Reihe von Gründen aufgeführt. 
sche Raum unter osmanischer Kontrolle 8 , und nach der Schlacht an der Marica (1371) war auch die serbische und bulgarische Selbständigkeit gebrochen. Von nun an gab es nur noch byzantinische Enklaven auf osmanischem Territorium. Hier liegt der geopolitische Ausgangspunkt, auf dem die ideologische Auseinandersetzung zwischen Osmanen und Byzantinern basierte.

\section{Allgemeine Überlegungen zur geistigen Auseinandersetzung}

Byzantinische Politik und Gesellschaft waren von Anbeginn an mit nomadischen oder halbnomadischen Völkern ebenso vertraut wie, seit dem 7. Jahrhundert, mit Stämmen und Staaten muslimischer Religion. ${ }^{9}$ Von dieser Ausgangsposition her erforderte der Umgang mit den Osmanen keine besondere neue Anpassung. Sie fügten sich gut in das seit Jahrhunderten geübte Muster für die Ungläubigen. Im Gegensatz zu den zahlreichen Streitschriften gegen christliche Häresien, zunächst gegen die Monophysiten in ihren verschiedenen Verzweigungen, dann seit dem 9. Jahrhundert vermehrt gegen die dogmatischen und liturgischen Abweichungen der römischen Kirche und die „Lateiner“ im allgemeinen, ist die Propaganda gegen die Muslime auf vergleichsweise wenige schriftliche Zeugnisse beschränkt. ${ }^{10}$ Dies sind zunächst mehr oder weniger ausführliche Passagen bei den Verfassern von Weltchroniken ${ }^{11}$, die sich auch (wenigstens teilweise) in Klosterbibliotheken fanden, und in Häresienverzeichnissen, die aber eher theoretischer Natur waren. ${ }^{12}$ In den Werken des Johannes von Damaskus und Theodoros von Abu Qurra, beide 8. Jahrhundert, sind Dialoge und Argumentationshilfen gegen die muslime Lehre überliefert, die auch eine handschriftlich weite Verbreitung besaßen. ${ }^{13}$ Erst Niketas Byzantios hat im 9. Jahrhundert einen ausführlicheren Traktat hinterlassen, auf den sich spätere Autoren wie Euthymios Zigabenos und Niketas Choniates stützten. ${ }^{14}$ In ihnen werden einzelne Koranpassagen untersucht, doch ist nicht zu vergessen, dass der

8 Beldiceanu 1965, 439-461.

9 Schreiner 1983, Šahīd 2010.

10 Eine frühe, wenig bekannte Untersuchung brachte Güterbock 1912. Auf einer gründlichen Kenntnis der islamischen Quellen beruht Eichner 1936, während die Übersicht bei Meyendorff 1964 ganz von byzantinischen Texten ausgeht.

11 Brandes 2009, 313-343 hat die Bedeutung der frühen Chronistik, im besonderes des Theophanes, für eine Kenntnis der Anfänge des Islam erstmals überzeugend herausgearbeitet.

12 Ein kurzer Überblick bei Trapp 1966, 14*-24*.

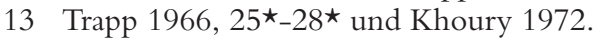

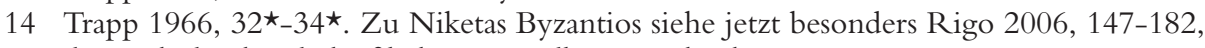
der auch den handschriftlichen Grundlagen nachgeht. 
Koran im byzantinischen Bereich immer nur in unzulänglichen Auszügen bekannt war. ${ }^{15}$ Die umfangreicheren Predigtcorpora, die allerdings bis heute überwiegend in philologisch unzulänglichen Ausgaben vorliegen, sind im Hinblick auf die Islampolemik bisher nicht untersucht. Alle Quellen, die uns bekannt und erhalten sind, entstanden fast ausnahmslos in Konstantinopel, wo bis ins 14. Jahrhundert der Islam keine politische und kaum eine ideologische Gefahr darstellte. Vielleicht gab es in den Grenzregionen zu muslimischen Staaten eine mündliche, propagandistische Auseinandersetzung, von der das Gespräch zwischen dem (christianisierten) Emir und seiner Mutter im Digenis Akritas-Epos noch Spuren zeigt. ${ }^{16}$ Einen schwachen und bescheidenen Einblick geben Abschwörungsformulare, die sich aber weder chronologisch noch topographisch festlegen lassen. ${ }^{17}$ Die stereotype Bezeichnung der Muslime als "gottlos, heidnisch, barbarisch“ in allen byzantinischen Quellengattungen ist zwar unter dem Stichwort „Propaganda" zu vermelden, hat aber mit einer intellektuellen Auseinandersetzung wenig zu tun.

\section{Die besondere Situation des 14. Jahrhunderts}

Erst die Bedrohung durch die Osmanen im 14. Jahrhundert, von der im zweiten Kapitel die Rede war, führte offensichtlich dazu, dass die intellektuelle Diskussion neu belebt wurde und uns zwei Texte überliefert sind, die an Informationsfülle und konkreter Überzeugungskraft die polemische Literatur der früheren Jahrhunderte weit übertreffen.

Noch vor diesen umfangreichen Texten liegen zwei Sendschreiben, die, wie zu zeigen ist, vom Inhalt her nur marginal in die muslimische Diskussion fallen, aber wegen der Hochrangigkeit der Persönlichkeit das Augenmerk der Öffentlichkeit wieder auf den ideologischen Gegner richteten. In den ersten Märztagen des Jahres 1354 wurde an den Dardanellen das Schiff eines der hochrangigsten byzantinischen Geistlichen, des Erzbischofs Gregorios Palamas von Thessaloniki, durch Osmanen gekapert. Gregorios war der repräsentativste Vertreter der neuen theologischen Richtung des Hesychasmus, der die orthodoxe Theologie der nächsten hundert Jahre und darüber hinaus entscheidend beeinflusste. Er

15 Trapp 1980 ist den Fragen der Koranübersetzung erstmals ausführlicher nachgegangen. Eine Sammlung der Fragmente (mit den zugehörigen Koransuren in deutscher Übersetzung) bringt Förstel 2009, 85-122. Eichner 1936, 438 war noch davon ausgegangen, dass Niketas Byzantios den Koran in der arabischen Originalsprache gekannt habe.

16 Hier zitiert nach der leicht zugänglichen (und auch übersetzten) Version von Grottaferrata, Buch III, vv. 111-245 (Digenis Akrites, ed. J. Mavrogordato. Oxford 1956).

17 Sahas 1991. 
verbrachte fast eineinhalb Jahre in Bursa und Nikaia in osmanischer Haft und verfasste in dieser Zeit drei Schriften. In einem Sendschreiben an seine Gemeinde schildert er zwar in erster Linie die Umstände seiner Gefangennahme, aber es fließen auch, wenngleich ohne Systematik, Diskussionen über Einzelfragen mit muslimischen Würdenträgern ein, etwa über die Person des Propheten oder über die Trinität und die Rolle Mariens. Dabei geht es immer um Einzelgespräche und noch nicht um eine umfassende Glaubensdiskussion. Dem prominenten Autor ist es zu verdanken, dass der Text des Schreibens erhalten blieb und in neun, zum Teil sogar jungen Handschriften des 15. und 16. Jahrhunderts vorliegt, die auf weiteres Interesse während der Turkokratie schließen lassen. Mit Sicherheit darf man annehmen, dass dieser Text den Gläubigen in der Diözese Thessalonike zur Kenntnis gelangte. ${ }^{18}$ Erhebliche Probleme bot und bietet der Forschung ein ebenfalls in neun Handschriften auf uns gekommenes Religionsgespräch desselben Gregorios Palamas - es trägt den Titel Dialexis - nicht mit Muslimen, sondern mit einer judaisierenden Sekte, die den bisher ungeklärten Namen „Chionai“ trägt. ${ }^{19}$ Ohne an dieser Stelle auf Einzelheiten einzugehen, handelt es sich um eine Apologie des christlichen Glaubens, der Kontroversfragen aufgreift, die der jüdischen und der muslimischen Theologie gleichermaßen eigen sind. ${ }^{20}$ Sendbriefe und Dialog sind die ersten byzantinischen Zeugnisse einer lebendigen und konkreten, auch historisch eindeutig greifbaren Auseinandersetzung mit der muslimischen Theologie.

Der tatsächliche Dialog mit muslimischen Gelehrten bei Gregorios Palamas zeigt, dass in der Diskussion mit Christen auch die muslimische Seite nicht unvorbereitet war. Diese Form der Diskussion mit Christen (und Juden) macht auch einen Teil der islamischen theologischen Literatur aus, ist aber außerhalb der islamwissenschaftlichen Fachdiskussion nie besonders zur Kenntnis genommen worden. ${ }^{21}$ Schon im 9. Jh. entstanden in den Territorien des „,christlichen Orients" muslimische Schriften gegen Melkiten, Jakobiten und Nestorianer. ${ }^{22}$ Sicher nicht ohne Grund ist aber die einflussreichste polemische Darstellung aus der Feder des Ibn Hazm (gest. 1064) im Spanien des 11. Jahrhunderts entstan-

18 Die Schreiben sind philologisch und inhaltlich von Philippidis-Braat 1979 erschlossen worden.

19 Text der Dialexis bei Philippidis-Braat 1979, 167-185.

20 In diesem Zusammenhang ist darauf hinzuweisen, dass der Judendialog ein traditionelles Genus der byzantinischen polemischen Literatur darstellt und wesentlich weiter verbreitet war als der Dialog mit den Muslimen, vgl. Külzer 1999.

21 Ich verdanke Anregungen und Hinweise zu diesem kurzen Abschnitt Tilman Nagel, Göttingen.

22 Ljamai 2003 in der Einführung zu seiner Monographie über Ibn Ḥam geht bibliographisch (besonders S. 1, Anm. 1) auf diese Autoren ein. 
den. ${ }^{23}$ Sie hat nicht nur in den späteren Kleinemiraten Spaniens nachgewirkt, sondern auch in den Ländern des von Christen bewohnten Kalifats. ${ }^{24} \mathrm{Hinzu-}$ weisen ist auch auf das Sendschreiben (Brief) des melkitischen Bischofs in Sidon, Paulos von Antiocheia, vom Beginn des 13. Jh. an muslimische Freunde in seiner Stadt $^{25}$, der rund 100 Jahre später in Cypern umgearbeitet und in dieser Form an muslimische Gelehrte in Damaskus geschickt wurde, wo er die Reaktion des hanbalischen Gelehrten Ibn Tayamiyya hervorrief. ${ }^{26}$

\section{Neue Grundlagen der Islampolemik}

Im selben Jahr 1354, als Gregorios Palamas in osmanischer Gefangenschaft weilte, vollendete Demetrios Kydones seine Übersetzung der Summa contra gentiles des Thomas von Aquin und begann jene der Improbatio Alcorani des Florentiner Predigers Riccoldo da Monte Croce (gest. 1320). ${ }^{27}$ Als Franziskanermissionar weilte dieser lange in Täbris und Bagdad und besaß auch eine sprachlich fundierte Korankenntnis. Die Übersetzung dieses Werkes des Franziskaners durch Kydones ist an sich schon bemerkenswert, wäre aber sicher wie andere Übersetzungen des ,lateinerfreundlichen“ Kydones kaum bekannt geworden ${ }^{28}$, hätte sie nicht Johannes Kantakuzenos als Grundlage und Ausgangspunkt seiner Apologien und Reden gegen Muhammad benutzt. Dieses Werk des ehemaligen Usurpators und Kaisers (Johannes VI.), der im Jahr 1354 zur Abdankung gezwungen wurde und bis zu seinem Tod (1383) historische und theologische Schriften verfasste, kann als das bedeutendste systematische Werk in der Islampolemik bezeichnet werden. ${ }^{29}$ Es beruht allerdings nicht, wie die Schriften seines hesychastischen Glaubensfreundes Palamas oder Kaiser Manuels II., von dem später die Rede sein wird, auf einem lebendigen Gespräch mit Muslimen, sondern ist die Antwort auf einen Brief des Persers Samprates aus Isfahan, den die moderne Forschung nicht mehr als fiktiv betrachtet. Das Werk entstand zwischen 1370 und 1374 und erfreute sich mit heute noch nachweisbaren 46 Handschriften bis

23 Ljamai 2003.Vgl. auch Behloul 2002.

24 Ljamai 2003, 175-196 (L'influence d'Ibn Hazm sur les savants postérieurs d'Orient).

25 Khoury 1994.

26 Diese Diskussion hat kürzlich Sarrió Cucarella 2009, 287-337 ausführlich behandelt.

27 Präzise Angaben zu seinem Leben im Lexikon des Mittelalters, Bd. 7. München 1995, 808 (C.-P. Haase). Zum Libellus (oder tractatus) contra Legem Saracenorum s. Todt 1991, 262-282.

28 Todt 1991, 298-301. Vollständige Quellen- und Literaturangaben zum Traktat des Riccoldo bei Tinnefeld 1981, 71 .

29 Die Schriften des Kantakuzenos gegen den Islam sind zugänglich in der Patrologia Graeca, Bd. 154, 371-692. Zur Interpretation s. Todt 1991, 306-566. 
weit in die postbyzantinische Zeit hinein größter Aufmerksamkeit. Dies lag an der Bedeutung des Autors nicht als (ehemaliger) Kaiser, sondern als Theologe, und an der Tatsache, dass dank des Traktates des Riccoldo eine aktuelle Basis für die antiislamische Polemik vorlag, die die Tradition der häretischen Kataloge verließ. Es ist aber zu betonen, dass ohne die ins Griechische übersetzten lateinischen Dokumente eine Diskussion, wie sie Johannes Kantakuzenos vorlegen konnte, nicht möglich gewesen wäre, auch wenn der politische Druck der Osmanen, der sich im 8. Jahrzehnt immer mehr auf Konstantinopel konzentrierte, der Schrift des Exkaisers große Aktualität verlieh. Erst durch den lateinischen Traktat des Riccoldo, der vor dem Hintergrund der scholastischen Theologie der Summa des Thomas von Aquin zu sehen ist, wurde auch die Diskussion in Byzanz auf eine sachlich fundierte Ebene gehoben. Diese letztlich von lateinischer Seite angeregte und ermöglichte Islamdiskussion ist ein bemerkenswertes Beispiel für den Einfluss der lateinischen scholastischen Methode auf die byzantinische Theologie in einer Zeit, in der in Byzanz selbst eher die antilateinische Polemik in Blüte stand.

\section{Die Schrift des Kaisers Manuel II. Palaiologos}

Auch das zweifellos theologisch anspruchsvollste Werk in der byzantinischen Islampolemik ist ohne die Apologie des Kantakuzenos nicht denkbar und stützt sich darauf auch inhaltlich. Es stammt aus der Feder Kaiser Manuels II. (13911425), mütterlicherseits Enkel des Kantakuzenos, und bedient sich nicht nur wieder der Dialogform, sondern ihr liegt auch eine konkrete Gesprächssituation während des Aufenthalts des Kaisers in Ankara als osmanischer Vasall in den letzten Monaten des Jahres 1391 zugrunde. ${ }^{30}$ Der Dialogtraktat setzt sich aus 20 Gesprächen zusammen, die an 20 aufeinander folgenden Tagen geführt wurden. ${ }^{31}$ Der Kaiser hatte einen Dolmetscher zur Verfugung (der auch manchmal erwähnt wird) und machte sich vermutlich tagebuchartige Aufzeichnungen. Sein Gesprächspartner ist ein islamischer Gelehrter, sekundiert bisweilen von dessen Söhnen, der als mudarris (im Griechischen als Eigenname „Muzarites“) bezeichnet wird, in dessen Haus sich der Kaiser aufhielt. Die Gespräche des in der klassischen griechischen Literatur hochgebildeten Kaisers folgen in ge-

30 Zum politischen Hintergrund siehe Barker 1969, 87-99.

31 Zu den Umständen der Entstehung des Dialogs s. Trapp 1966, 54^-58*. Das Werk wurde erstmals vollständig von Trapp 1966, 2-202 herausgegeben. Dieser griechische Text liegt der deutsch-griechischen Ausgabe von Förstel 1993-1996 zugrunde. Offensichtlich ohne Kenntnis dieser Übersetzung hat Senoner 2003 die Dialoge 1, 2, 5, 6 und 7 nochmals ins Deutsche übertragen. 
wissem Sinn der Form von Platons Dialogen, wenngleich diese den Tag nie überschreiten und innerhalb einer zeitlichen Einheit auch eine philosophische Thematik abschließen.

Im Gegensatz zu den Traktaten des Kantakuzenos stellen diese Dialoge doch ein recht elitäres und isoliertes Werk dar, zu dem die hochrhetorische Sprache des Kaisers noch ihren Teil beiträgt. Der Text ist Manuels Bruder Theodoros, der 1407 starb, gewidmet, doch spricht vieles dafür, dass die Abfassung schon 1392 erfolgte. Er ist nur in vier Exemplaren erhalten, von denen drei nur Abschriften aus dem 16. und 18. Jahrhundert sind. Die Dialoge hatten also, über den Adressaten hinaus, keinen weiteren Leserkreis, und fanden als Ganzes erst vor einem halben Jahrhundert einen Editor.

Auch die fingierte Dialexis eines Zeitgenossen des Kaisers, des Joseph Bryennios, der durch eine Fülle von theologischen Werken keinen unbedeutenden Platz in der byzantinischen Literatur einnimmt, ist nur in zwei Handschriften, darunter eine aus dem 18. Jahrhundert, überliefert, die bis jetzt unediert blieben. ${ }^{32}$ Es zeigt sich auch hier, dass in den Jahrzehnten stärkster osmanischer Bedrohung kein größeres Interesse an einer theoretischen Diskussion bestand, ganz im Gegensatz zur Lateinerpolemik, die wegen der Unionsverhandlungen weiterhin eine zentrale Rolle spielte.

\section{Der Außenseiter}

Weder Palamas noch Kaiser Manuel hatten sich den Diskussionen freiwillig gestellt, und der Aufenthalt im osmanischen Reich vollzog sich mehr oder weniger unter Zwang. Dagegen hielt sich einer der größten byzantinischen Gelehrten, Georgios Gemistos (der sich nach 1438 Plethon nannte) freiwillig, der Ausbildung wegen, am Hof des Sultan in Adrianopel (kaum in Bursa) auf. ${ }^{33}$ Wir wissen darüber nur aus einigen Passagen in den Schriften seines später härtesten Gegners, des Patriarchen Gennadios Scholarios. Der Aufenthalt, der wohl zwischen 1380 und 1390 anzusetzen ist, hatte aber mit der islamischen Kultur allenfalls nur am Rande zu tun, vielmehr galt er der Ausbildung beim jüdischen Leibarzt des Sultans, Elisäus, der den etwa zwanzigjährigen Georgios Gemistos mit der antiken Philosophie vertraut machte. Die Forschung hat in verschiedenen Passagen seiner Schriften (etwa beim Begriff der Vorsehung) einen Einfluss der Lehre des

32 Trapp 1966, 91*-93*.

33 Die klassische Untersuchung hat Masai 1956 verfasst, neue Gesichtspunkte bringen Woodhouse 1986 und Tambrun 2006, 9-33 sowie jüngst Carabă (2010) bei. 
Koran feststellen wollen. ${ }^{34}$ Eine minutiöse Untersuchung, die schon vor mehr als einem halben Jahrhundert Milton Anastos vornahm, zeigte jedoch, dass Ideen in dieser Richtung auch von der byzantinischen Philosophie und Theologie her erklärt werden können ${ }^{35}$, während eine jüngst erschienene Studie unter $\mathrm{Zu}$ grundelegung arabischer Quellen arabisch-muslimischen Einfluss durchaus für denkbar hält. ${ }^{36}$ Den Makel eines „Sarakenophron“ wurde Plethon freilich in den Augen der Orthodoxen nicht los. ${ }^{37}$ Seine Distanz zur byzantinischen Schultradition und sein rigoroser Rückgriff auf die „heidnische“ griechische Antike, derentwegen er als ,letzter der Hellenen“ bezeichnet wird ${ }^{38}$, verdankt er in hohem Umfang der geistigen Welt seines jüdischen Lehrers und einer intellektuellen Neutralität, wie sie der byzantinische Kaiserhof schon lange nicht mehr bot, für einen „Heiden“ wie Plethon aber vielleicht eher der Sitz des Sultans. Die Person Plethons, der als Konzilsteilnehmer immer die Wertschätzung der orthodoxen (und der lateinischen) Kirche genoss, ist wohl am ehesten im Sinne einer inneren geistigen Auseinandersetzung mit den Osmanen in diese Darstellung mit einzubeziehen.

\section{Polemik ohne Breitenwirkung}

Es besteht kein Zweifel, dass der theologischen Diskussion gegen den Islam jede Breitenwirkung fehlt, und dass gerade in den letzten Jahrzehnten des byzantinischen Staates dem Protest gegen Lehre und Einfluss der römischen Kirche und der Politik westlicher Nationen im Allgemeinen wesentlich größere Bedeutung zukam. Die byzantinischen Historiker bezeichnen zwar in traditioneller Weise die Osmanen als „Barbaren“. Man hat aber durchaus den Eindruck, dass Kaiser Manuel seinen muslimischen Gesprächskontrahenten als intellektuell adäquaten Gegner betrachtete. Die Verbreitung von persischem und arabischem Wissen am Hof der Sultane schon vor der Eroberung Konstantinopels war byzantini-

34 Besonders Taeschner 1929, doch hat schon Franz Dölger in einer Kurzrezension diese Ansicht zurückgewiesen (vgl. Byzantinische Zeitschrift 31, 1931, 126), s.a. Carabă 2011.

35 Anastos 1948, 270-305

36 Akasoy 2003, 339-353. So auch Carabă 2011.

37 „Sarakenophron“ (man denkt wie ein Muslim) begegnet erstmals in der Chronik des Theophanes Confessor (ed. de Boor S. 339) und war neben „latinophron“ ein häufig angewandter Ausdruck der Diffamierung. Der Begriff verdiente eine eigene Untersuchung, die die Bedeutung der Muslime und ihrer „Sympathisanten“ im byzantinischen Pamphlet aufzeigen könnte.

38 Es ist mir nicht gelungen, eine Belegstelle für dieses Dictum zu finden. Woodhouse 1986 verwendet es ohne Quellenangabe S. 379 und bezieht sich auf Zakythinos 1953, 350: „,en ce sens, Pléthon mérite d'être considéré comme le dernier des Byzantins et le premier des Néo-hellènes“. Dagegen hätte Plethon sicher heftig protestiert! 
schen Hofkreisen schwerlich verborgen geblieben. Der byzantinische Historiker Dukas überliefert, nach Abschluss der unbeliebten Kirchenunion im Dezember 1452 den Ausspruch des Lukas Notaras, des höchsten Hofbeamten: „Es ist besser, in Konstantinopel einen türkischen Turban herrschen zu sehen als eine lateinische Kaiserkrone. “39 Das Dictum ist häufig falsch übersetzt und interpretiert worden (indem man an die Stelle der Kaiserkrone die päpstliche Tiara setze und den Ausspruch auf die Glaubensdifferenzen bezog). ${ }^{40}$ Vielleicht ist es eher dem Historiker selbst als dem hohen Hofbeamten (der nach 1453 vom Sultan hingerichtet wurde) zuzuschreiben. Es zeigt sehr deutlich eine in Konstantinopel, das nun territorial fast identisch mit dem Reich war, herrschende Stimmung, die in den Osmanen nicht mehr unbedingt einen oder den alleinigen Feind sah, und es lässt sich die Frage nicht ganz unterdrücken, ob es wirklich eine ernsthafte geistige Auseinandersetzungen gegen den Islam je gegeben hat.

Das emphatische Widmungsschreiben an Sultan Mehmed, das einer der letzten byzantinischen Geschichtsschreiber, Laonikos von Imbros, seinem Werk voransetzte $^{41}$, lässt daran ebenso zweifeln wie die Haltung der in Konstantinopel verbliebenen Schicht griechischer Intellektueller, die in Mehmed zwar einen Ungläubigen sahen, der jedoch von der theologischen Haltung her dem Christentum Verständnis entgegenbrachte und die hellenischen Wissenschaften nicht weniger förderte als einst der byzantinische Kaiser. ${ }^{42}$ Als das byzantinische Reich nicht mehr existierte und auch die geistige Auseinandersetzung zwischen Griechen und Muslims mildere Töne anschlagen musste, war in gewissem Sinn ein Ausgleich erreicht.

\section{Literaturverzeichnis}

(Stand Mai 2012)

Akasoy, Anna, 2003: „George Gemistos Pletho and Islam“, in: Proceedings of the International Congress of Plethon and his time, Mystras 26-29 June 2002. Athen-Mistras, 339-353.

Anastos, Milton, 1948: „Plethons Calendar and Liturgy“, in: Dumbarton Oaks Papers 4 (1948) 183-305.

Barker, John W., 1969: Manuel Palaiologus (1391-1425): A Study in Late Byzantine Statemanship. New Brunswick.

39 Grecu 1958, 329, 11-12.

40 Die sprachlich richtige Interpretation und Übersetzung bei Reinsch 1996, 377-389.

41 Reinsch 1983, 3-9. Deutsche Übersetzung Reinsch 1986, 33-37.

42 Schreiner 2009, 31-40. Zur theologischen Einstellung des Patriarchats ausfuihrlich Vryonis 1996, der hier auch zur Haltung des Patriarchats gegenüber Muslim-Türken in früheren Jahrhunderten Stellung nimmt. 
Behloul, Samuel-Martin, 2002: Ibn Hazms Evangelienkritik. Eine methodische Untersuchung. Leiden.

Beldiceanu, Irène, 1965: „La conquête d'Adrianople par les Turcs: la pénétration turque en Thrace et la valeur des chroniques ottomans“, in: Travaux et Mémoires 1 (1965), 439-461.

Benedikt XVI, 2006: Benedikt XVI., Glaube und Vernunft. Die Regensburger Vorlesung. Vollständige Ausgabe (kommentiert von Gesine Schwan, Adel Theodor Khury, Karl Kardinal Lehmann). Freiburg.

Brandes, Wolfram, 2009: „Der frühe Islam in der byzantinischen Historiographie. Anmerkungen zur Quellenproblematik der Chronographia des Theophanes“, in: Andreas Goltz u. a. (Hrsg.): Jenseits der Grenzen. Beiträge zur spätantiken und frühmittelalterlichen Geschichtsschreibung. Berlin.

Carabă, Vasile A., 2010: Pletho Apostata: die Ablehnung des Christentums durch Georgios Gemistos Plethon (ca. 1355-1452) und dessen Konversion zur griechischen Religion. Gießen.

Carabă, Vasile A., 2011: „What is known about Elissaeus (14 ${ }^{\text {th }}$ century), a Teacher of Georgios Gemistos Plethon ( ${ }^{\star}$ ca.1355-†1452)?“", in: Études Byzantines et PostByzantines, 6 (2011), 170-185.

Demetracopoulos, J. A., 2008: „Pope Benedicts XVI' use of the Byzantine emperor Manuel II Palaiologos Dialogue with a Muslim muterizes: the scholary background“", in: Archiv für mittelalterliche Philosophie und Kultur 14 (Sofia 2008), 264-304.

Eichner, W., 1936: „Die Nachrichten über den Islam bei den Byzantinern“, in: Der Islam 23 (1936) 133-162, 197-244.

Förstel, Karl, 1993-1996: Manuel II. Palaiologos. Dialoge mit einem Muslim. Kommentierte griechisch-deutsche Textausgabe von Karl Förstel. 3 Bde. Würzburg.

Förstel, Karl, 2009: Schriften zum Islam von Arethas und Euthymios Zigabenos und Fragmente der griechischen Koranübersetzung. Wiesbaden.

Grecu, Vasile, 1958: Ducas. Istoria turco-bizantina(1341-1462), ed. V. Grecu. Bukarest.

Güterbock, Carl, 1912: Der Islam im Lichte der byzantinischen Polemik. Berlin.

Hopwood, K., 1992: „Low-level Diplomacy between Byzantines and Ottoman Turks: the Case of Bithynia“, in: Shepard, Jonathan / Franklin, Simon: Byzantine Diplomacy. Aldershot.

Inalcik, Halil, 1993: „Osmān Ghāzī’s siege of Nicea and the battle of Bapheus“, in: Zachariadou, Elizabeth: The Ottoman Emirate (1300-1389). Rhethymnon.

Karpozilos A., 1970: „Byzantine Apologetic and Polemic Writings of the Palaeologian Epoch against Islam“, in: Greek Orthodox Theological Review 15 (1970), 213-248.

Khoury, Adel-Théodore, 1972: Polémique byzantine contre l'Islam (VIII-XIII s.). Leiden.

Khoury, Paul, 1994: Paul d'Antioche. Traités théologiques. Würzburg.

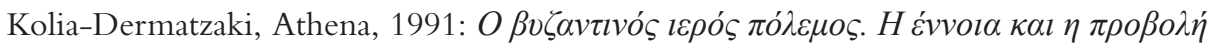

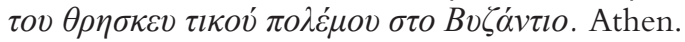

Külzer, Andreas, 1999: Disputationes graecae contra Iudaeos. Untersuchungen zur byzantinischen antijüdischen Dialogliteratur und ihrem Judenbild. Stuttgart. 
Ljamai, Abdelilah, 2003: Ibn Hazm et la polémique islamo-chrétienne dans l'histoire de l'Islam. Leiden.

Luthe, Heinz-Otto / Walbiner, Carsten-Michael, 2008: Anstoß und Aufbruch. Zur Rezeption der Regensburger Rede Papst Benedikts XVI. bei Christen und Muslimen. Bochum.

Masai, François, 1956: Pléthon et le platonisme de Mistra. Paris.

Meyendorff, John, 1964: „Byzantine Views of Islam“, in: Dumbarton Oaks Papers 18 (1964), 113-132.

Philippidis-Braat, Anna, 1979: „La captivité de Palamas chez les Turcs: dossier et commentaire“, in: Travaux et Mémoires 7 (1979), 109-121.

Reinsch, Diether R., 1983: Critobuli Imbriotae Historia, rec. Diether Roderich Reinsch. Berlin.

Reinsch, Diether R., 1986: Mehmed II. erobert Konstantinopel. Die ersten Regierungsjahre des Sultan Mehmet Fatih, des Eroberers von Konstantinopel 1453. Das Geschichtswerk des Kritobulos von Imbros (übersetzt, eingeleitet und erklärt von Diether Roderich Reinsch). Graz.

Reinsch, Diether R., 1996: „Lieber der Turban oder was?“, in: $\Phi_{\imath} \lambda \dot{\lambda} \lambda \lambda \eta v$. Studies in honour of Robert Browning. Venedig.

Rigo, Antonio, 2006: „Niceta Byzantios, la sua opera e il monaco Evodio“, in: Fiaccadori, Gianfranco (Hrsg.): „In partibus Clius“. Scritti in onore di Giovanni Pugliese Caratelli. Neapel.

Sahas, D. J., 1991: „Ritual Conversion from Islam to Byzantine Church“, in: Greek Orthodox Theological Review 3 (1991), 57-69.

Šahīd, 'Irfān, 2010: Byzantium and the Arabs in the Sixth Century, vol. II, 2. Economic, social and cultural history. Washington.

Sarrió Cucarella, Diego R., 2009: „Un irreductible choque de orthodoxias: Ibn Taymiyya y la controversia religiosa medieval en torno a la interpretación del Corán“, in: Collectania Christiana Orientalia 8 (2009), 287-337.

Schreiner, Peter, 2009: „Die Epoche Mehmets des Eroberers in zeitgenössischen Quellen aus dem Patriarchat", in: Asutay-Effenberger Neslihan / Rehm Ulrich (Hrsg.): Sultan Mehmed II. Eroberer Konstantinopels - Patron der Künste. Köln, 31-40.

Senoner, Raimund, 2003: Kaiser Manuel II. Palaiologos: Dialog über den Islam und Erziehungsratschläge. Mit drei Briefen König Sigismunds von Luxemburg an Manuel II. (Übersetzung: Raimund Senoner, herausgegeben und eingeleitet von Wilhelm Baum). Klagenfurt.

Stouraitis, Ioannis, 2009: Krieg und Frieden in der politischen und ideologischen Wahrnehmung in Byzanz (7.-11. Jahrhundert). Wien.

Taeschner, Franz, 1929: „Georgios Gemistos Plethon. Ein Beitrag zur Frage der Übertragung von islamischen Geistesgut nach dem Abendlande“, in: Der Islam 18 (1929), 236-240.

Tambrun, Brigitte, 2006: Pléthon. Le retour de Platon. Paris.

Tinnefeld, Franz, 1981: Demetrios Kydones. Briefe. (übersetzt und erläutert von Franz Tinnefeld). Bd. I,1. Stuttgart. 
Todt, Klaus-Peter,1991: Kaiser Johannes VI. Kantakuzenos und der Islam. Politische Realität und theologische Polemik im palaiologenzeitlichen Byzanz. Würzburg.

Trapp, Erich, 1966: Manuel Palaiologos. Dialoge mit einem „Perser“. Wien.

Trapp, Erich, 1980: „Gab es eine byzantinische Koranübersetzung?“”, in: Diptycha 2 (1980/81), 7-17.

Vryonis, Spiros, 1996: „The Byzantine Patriarchate and Turkish Islam“, in: Byzantinoslavica 57 (1996), 69-111.

Werner, Ernst, 1966: Die Geburt einer Großmacht-Die Osmanen. Wien.

Woodhouse, Christopher M., 1986: George Gemistos Plethon. The Last of the Hellenes. Oxford.

Zakythinos, Denys A., 1953: Le despotat grec de Morée. Bd. 2. Athen. 